\section{Hysterosalpingographic findings in primary and secondary infertility patients}

\author{
Khawaja B. Waheed, MBBS, FCPS, \\ Muneera A. Albassam, MBBS, JB-RAD (Jordanian Board), \\ Alaa Ali G. AlShamrani, MBBS, SB-RAD (Saudi Board), \\ Shayma A. Aloumi, MBBS, SB-RAD (Saudi Board), \\ Muhammad S. Amin, MBBS, MCPS, \\ Lubna Rashid, $M B B S, M D$, \\ Sarah M. AlGodayan, GSCE, MBBS, \\ Siti Fatimah B. Ahmad, SPM, BSC.
}

\section{ABSTRACT}

Objectives: To highlight and compare spectrum of hysterosalpingography (HSG) findings in primary and secondary infertility patients.

Methods: This retrospective record-based crosssectional study was performed in the Radiology Department, King Fahad Military Medical City, Dhahran, Kingdom of Saudi Arabia between August 2016 and 2018. All patients $(\mathrm{N}=303)$ who underwent successful HSGs were included, and grouped under primary and secondary infertility cases. Patients with failed, limited or incomplete studies were excluded. Imaging findings were documented as $\mathrm{N}$ (Normal) or Ab (Abnormal). Abnormal HSG findings were further categorized as: $\mathrm{C}=$ congenital malformation, $\mathrm{I}=$ infection or inflammation, $\mathrm{S}=$ surgery, $\mathrm{T}=$ tumor or tear. Abnormal findings were confirmed on further imaging or intervention. Chi-square test was used to determine any association of HSG findings with type of infertility, and $p$-value less than 0.05 was considered significant.

Results: Of the 303 patients, 166 patients (54.8\%) had primary infertility while the rest had secondary infertility. Abnormal studies were found in less than one-third of patients $(n=93,30.7 \%)$. Primary infertility patients exhibited more congenital (C) malformations, while surgery $(S)$ was seen more in secondary infertility patients $(p=0.01)$.

Conclusion: Congenital malformations are commonly found abnormalities on HSGs in primary infertility patients, while surgery related findings are seen in secondary infertility cases.

Saudi Med J 2019; Vol. 40 (10): 1067-1071 doi: 10.15537/smj.2019.10.24538

Tnfertility, failure of a couple to conceive for at least 1 one year after an unprotected and regular sexual intercourse, affects approximately $15 \%$ of couples. ${ }^{1}$
Infertility can be primary (never conceived) and secondary (conceived earlier but failed to conceive afterwards). Uterine abnormalities (15\%), ovulatory dysfunction (20-40\%) and tubo-peritoneal pathologies (30-40\%) account for female factors of infertility. ${ }^{2}$ There has been an increase in number of women seeking infertility evaluation because of greater concern and awareness about this condition. Hysterosalpingography (HSG) remains a valuable screening procedure in the initial workup of infertility. ${ }^{3}$ Hysterosalpingography or uterosalpingography, is an $\mathrm{x}$-ray fluoroscopy imaging procedure that is used to delineate uterus and fallopian tubes by administering radio-opaque contrast material through a uterine catheter or cannula. Prior patient counselling, use of premedication, ${ }^{4}$ and adopting a better injection technique help to avoid or minimize technical artifacts and procedural complications. The results of HSG studies have been shown to be important for selecting patients for further imaging or intervention. ${ }^{5}$ A few patients may even conceive after this procedure, signifying its therapeutic benefits. ${ }^{6,7}$ Although various studies in literature have demonstrated role of HSG for infertility work-up, and its comparison with other diagnostic options; $;^{7,8}$ however, findings on this imaging have never been categorized and compared between the 2 types (primary and secondary) of infertility cases. Therefore, we aim to highlight and compare imaging findings in such groups.

Methods. This retrospective record-based crosssectional study was conducted in the Radiology Department, King Fahad Military Medical City, Dhahran, Kingdom of Saudi Arabia between August 2016 and 2018. All patients $(N=303)$ who underwent successful HSGs were included, and grouped under primary and secondary infertility cases. Patients with failed, limited or incomplete studies were excluded. Because the study was retrospective and did not involve disclosure of any patient information and privacy, the ethics committee of our hospital waived the need for patient consent. The study was conducted in accordance with the Helsinki Declaration.

All clinical and radiologic information were kept strictly confidential. A literature review was performed using an electronic search (Google Scholar, PubMed). Demographic information regarding age of all patients was collected. Clinical information and further test results were acquired through patients' clinical notes, Hospital Information System (HIS), Radiology Information System (RIS), and Picture Archiving and Communication System (PACS).

All HSG studies were performed by the radiologist with the assistance of nurse and radiographer, under 
fluoroscopy (C-arm, Siemens 2010, Artis Zee, Munich, Germany). Water-soluble iodinated contrast medium (Omnipaque, Iohexol, GE Healthcare, Cork, Ireland) was used for injection. Spot films were obtained for early and complete filling of the uterine cavity, delineation of fallopian tubes, and at time of contrast spillage into the peritoneal cavity.

Imaging findings were interpreted by 2 general radiologists (each having special interest in women imaging and having more than 7 years of experience), and consensus reporting was made for imaging diagnoses. Imaging findings were documented as $\mathrm{N}$ (Normal; normal uterine cavity and patent salpinges) or Ab (Abnormal; uterine or tubal abnormalities). Abnormal HSG findings were further categorized in to: $\mathrm{C}=$ congenital malformation (namely, unicornuate, bicornuate, septate, uterine didelphys), I=infection or inflammation (namely, salpingitis isthmica nodosa, hydrosalpinx, inflammatory peritubal adhesions), $S$ =surgery (namely, salpingectomy, excision of ovarian cyst or tubal ectopic and associated post-surgical adhesions), $\mathrm{T}$ =tumor or tear (namely, mass, polyp, fibroid, adenomyosis). Abnormal findings were confirmed on either further imaging (transvaginal ultrasound or MRI), or intervention (hysteroscopy or laparoscopy).

The statistical analysis was carried out using Statistical Package for Social Sciences (SPSS Statistics for Windows, version 22; IBM Corp., Armonk, NY, USA). Chi-square test was used to determine association of HSG findings with type of infertility, and $p$-value less than 0.05 was considered significant.

Results. Of the 303 patients, 166 patients (54.8\%) had primary infertility and rest had secondary infertility. The mean age was 32.35 (standard deviation [SD],
5.8 years). Abnormal studies were found in less than one-third of patients ( $\mathrm{n}=93,30.7 \%$ ).

Figure 1 summarized the normal and abnormal HSG findings. Figure 2 summarized the selected images of 2 patients (A \& B) demonstrating abnormal HSG findings confirmed on subsequent MRI.

Abnormalities in both primary and secondary infertility patients were segregated (Table 1). Primary infertility patients exhibited more congenital malformations $(\mathrm{C})$, while surgery $(S)$ related causes were seen more in secondary infertility patients $(p=0.01)$.

Findings pertaining to infection (I) commonly included hydrosalpinx (either unilateral or bilateral) in 14 out of 17 primary infertility patients, and in 18 out of 19 secondary infertility patients.

Two patients in primary and one in secondary had peri-tubal adhesions and contained collections with prior histories of pelvic inflammatory diseases (PID). One patient in primary was found to have salpingitis isthmica nodosa (SIN), confirmed on subsequent (MR) imaging. Congenital anomalies (C) were found to be unicornuate $(n=7)$, completely septate $(n=3)$, and uterine didelphys $(\mathrm{n}=1)$ in primary infertility patients, while a bicornuate and a partial septate among secondary infertility patients. Out of 3 cases of surgery, 2 patients had unilateral ovarian cystectomies and one had unilateral salpingectomy (complicated PID) among primary infertility cases. Whereas, among 9 cases of surgery in secondary infertility cases, 4 cases had unilateral salpingectomies (for ectopic gestations), 3 had ovarian cystectomies ( 2 unilateral, 1 bilateral), 1 had complicated cesarean section and one had myomectomy. Eleven cases in primary and 15 cases in secondary had fibroids (submucosal or mural), while

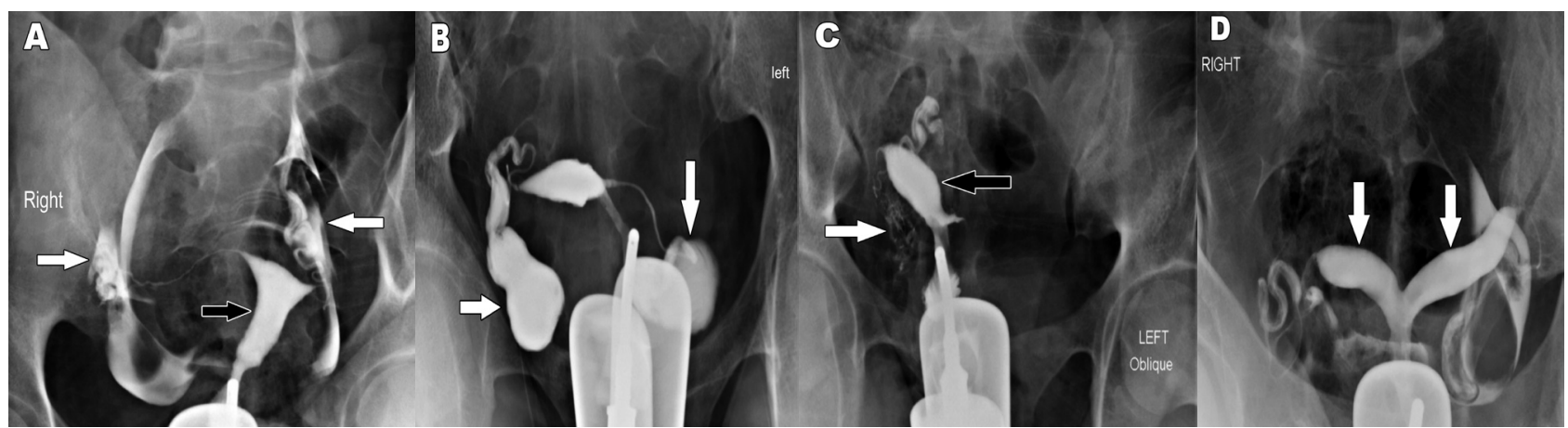

Figure 1 - Normal and abnormal hysterosalpingography (HSG) findings A) Normal study; normal uterine shape and contours (black arrow) with patent salpinges (white arrows) in a primary infertility patient. B) Bilateral dilated and blocked tubes/hydrosalpinx (white arrows) in a primary infertility patient. C) Unicornuate uterus (black arrow) in a secondary infertility patient. Note mild right sided intravasation (white arrow). D) Bicornuate uterus in a secondary infertility patient with 2 separate uterine horns (white arrows). 

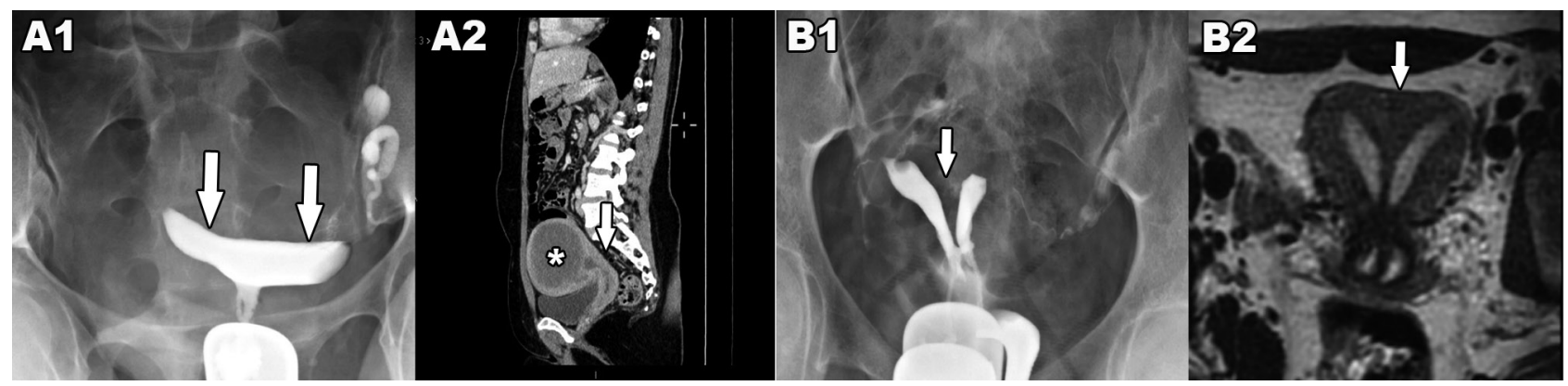

Figure 2 - Selected images of 2 patients (A \& B) demonstrating abnormal hysterosalpingography (HSG) findings confirmed on subsequent MRI: A1- Flattened contour of uterine cavity is seen on HSG in a secondary infertility patient. A2- Selected sagittal reformat CT image of same patient showing a large degenerated fundal fibroid (star) compressing upon the uterine cavity (arrow). B1- Suspicion of septate uterus with two uterine cavities (arrow)on HSG in a primary infertility patient. B2- Selected T2W coronal reformat image of the same patient showing completely septate uterus with myometrial tissue between the 2 uterine horns (arrow).

Table 1 - Percentage distribution of hysterosalpingography (HSG) findings in primary and secondary infertility.

\begin{tabular}{lcr}
\hline HSG findings & \multicolumn{2}{c}{ Infertility } \\
& Primary & Secondary \\
\hline Normal & $122(73.5)$ & $88(64.2)$ \\
Congenital & $11(6.6)$ & $2(1.46)$ \\
Infection or & $17(10.2)$ & $19(13.8)$ \\
inflammation & & \\
Tumor or tear & $13(7.8)$ & $19(13.8)$ \\
Surgery & $3(1.8)$ & $9(6.57)$ \\
Total & $166(100)$ & $137(100)$ \\
\hline \multicolumn{3}{c}{} \\
\hline
\end{tabular}

2 cases in primary and 4 cases in secondary infertility had infertility had endometriosis (adenomyosis, endometrioma).

Discussion. Hysterosalpingography is a commonly performed investigation for initial work up of infertility, ${ }^{5}$ providing both structural information of the uterine cavity and patency of the fallopian tubes. Al-Turki et $\mathrm{al}^{2}$ presented results of 5 years data from a University Hospital in Khobar, and demonstrated that $80 \%$ of abnormalities detected on HSG involved the tubes, while $20 \%$ were related to uterus. We categorized HSG findings based on etiology, and focused upon prevalence of these among the primary and secondary infertility patients. Such division of patients is not only of clinical relevance (in terms of patients' presentation, approach and counselling) but also seemed to have more specific etiological trend (and radiologic findings). More congenital malformations were observed in primary infertility patients, while more surgery related abnormalities were seen in secondary infertility patients; these results were found statistically significant $(p=0.01)$ (Table 1$)$. Although statically not significant, relatively more normal (HSG) studies in primary infertility cases may have clinical significance depicting other contributory factors towards infertility in these patients such as hormonal, psycho-sexual and social reasons. Statistically significant surgery related causes in secondary infertility patients were likely linked to their previous pregnancies and potentially associated surgeries (either for ectopic or cesarian sections). Although tumor/tear related causes were not seen statistically significant, their increased percentage in secondary infertility patients were also attributable to their already activated hormonal/ reproductive status and child birth or abortion. Also, slightly more infection related causes in secondary infertility patients might also be attributed to relatively more surgeries (either caesarian sections or related to ectopic pregnancies) in this group.

We used water soluble contrast for the the HSG

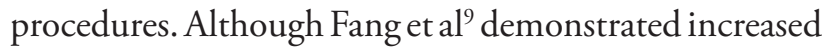
pregnancy rates after the use of oil-based contrast for tubal injections. However, prospective cohort study by Dreyer et $\mathrm{al}^{10}$ showed that probability of natural conception within 24 hours after first presentation at fertility clinic was increased after HSG regardless of type of contrast medium used, compared to patients having no HSG performed. We did not estimate pregnancy rates among our study patients, as such evaluation may not be that simple (considering variability of HSG findings, any abnormalities detected, their specific management options and outcomes, compliance to treatment, hormone status, and even quality of sexual relationships), and also require multivariate analysis.

An important aspect of HSG examination is to identify Mullerian duct anomalies, as few of these 
may be associated with infertility. ${ }^{11}$ We observed more of such anomalies in primary infertility patients. Differentiation of certain entities such as septate and bicornuate uterus can be sometimes difficult on HSG study, and may require further imaging such as 3-dimensional sonography or MRI for confirmation (Figure 2). Recognition is important as septate uterus is usually associated with early abortions and infertility, and may require surgery (excision of septum). ${ }^{12}$ Ludwin et $\mathrm{a}^{13}$ estimated indentation depth, indentation angle and indentation-to-wall-thickness (I:WT) ratio for identifying septate uterus. They compared diagnostic criteria by European Society of Human Reproduction and Embryology (ESHRE)-European Society for Gynaecological Endoscopy (ESGE) (I:WT ratio $>50 \%$ ) and American Society for Reproductive Medicine (ASRM) (depth $>15 \mathrm{~mm}$ and angle $<90^{\circ}$ ). They suggested that ESHRE-ESGE cut-off value overestimated the prevalence of septate uterus while that of ASRM underestimated that prevalence, and recommended considering indentation depth $\geq 10 \mathrm{~mm}$ as septate, since the measurement was simple and reliable and the criteria was seen in agreement with expert opinion. We used the same criteria to correctly identify septate uterus in 3 of primary infertility patients, that were confirmed on subsequent MRI. One of these underwent hysteroscopic septum resection. However, more recently Rikken et $\mathrm{al}^{12}$ did not find improved reproductive outcome to support such surgical procedure in these women, and stressed upon need of randomized controlled trials to validate safety of these procedures. ${ }^{12}$

Presently, techniques like sonohysterosalpingography (or sonohysterography) and magnetic resonance hysterosalpingography (MR-HSG) have also been evaluated as imaging options other than conventional hysterosalpingography (X-HSG) and 3-D CT-hysterosalpingography (CT-HSG], to avoid radiation risks. ${ }^{14,15}$ Although sono-HSG may have an advantage over X-HSG in not utilizing radiation; however, Maheux-Lacroix et al observed a $26 \%$ pregnancy rate in HSG group compared to $15 \%$ in sono-HSG while retrospectively evaluating outcome in 440 infertile women. ${ }^{7}$ Volondat et $\mathrm{al}^{14}$ demonstrated that MR-HSG was a well-tolerated technique demonstrating high accuracy in investigating tubal patency and intra-uterine abnormalities for diagnostic work-up of female infertility. Bhatt et $\mathrm{al}^{15}$ found that 3D CT-HSG could detect various factors responsible for female infertility in cases where HSG failed to clearly delineate the pathology. We feel that availability of such imaging options is certainly helpful to cater different patient strata, their conditions and preferences, and to overcome limitations of some modalities.

Study limitations. We consider small sample size, single center, retrospective and limited data study to be few limitations of our study. Selection of patients for other imaging options based on their abnormal HSG findings needs to be considered in the infertility clinic assessment pathway to reach early diagnosis and management. Larger prospective studies are needed to compare feasibility of such approach and to validate justification of combined or complimentary imaging in selected patients.

In conclusion, congenital malformations and surgery related causes are commonly found HSG findings in primary and secondary infertility patients respectively.

Acknowledgment We would like to thank our colleague Dr. Muneera A. Albassam for providing her assistance in reviewing the submitted figures for clarity and correctness.

Received 25th May 2019. Accepted 2nd September 2019.

From the Radiology Department, King Fahad Military Medical City, Dhahran, Kingdom of Saudi Arabia.

Address correspondence and reprints request to: Dr. Khawaja Bilal Waheed, Department of Radiology, King Fahad Military Medical Complex, Dhahran, Kingdom of Saudi Arabia. E-mail: Khawaja@kfmmc.med.sa

ORCID ID: https://orcid.org/0000-0001-6340-3139

\section{References}

1. Zegers-Hochschild F, Adamson GD, Dyer S, Racowsky C, de Mouzon J, Sokol R, et al. The International Glossary on Infertility and Fertility Care, 2017. Fertil Steril 2017; 108: 393-406.

2. Al-Turki HA, Gullenpet AH, Syed A, Al-Saif HS, Aldhafery BF. Uterine and tubal abnormalities in infertile Saudi Arabian women: A teaching hospital experience. Saudi J Med Med Sci 2016; 4: 89-92.

3. Onwuchekwa CR, Oriji VK. Hysterosalpingographic (HSG) Pattern of Infertility in Women of Reproductive Age. J Hum Reprod Sci 2017; 10: 178-184.

4. Jitchanwichai A, Soonthornpun K. Effect of premedication hyoscine-n-butylbromide before hysterosalpingography for diagnosis of proximal tubal obstruction in infertile women: A randomized double-blind controlled trial. J Minim Invasive Gynecol 2019; 26: 110-116.

5. Lopes VM, Barguil JP, Lacerda TS, Souza AL, Rocha Filho AM, Roller MF, et al. An overview of the results of hysterosonography prior to in vitro fertilization. JBRA Assist Reprod 2017; 21: 302-305.

6. Martí-Bonmatí L. Hysterosalpingography and fertility: a technical relationship. Fertil Steril 2018; 110: 642.

7. Maheux-Lacroix S, Bergeron C, Moore L, Bergeron MÈ, Lefebvre J, Grenier-Ouellette I, et al. Hysterosalpingosonography is not as effective as hysterosalpingography to increase chances of pregnancy. J Obstet Gynaecol Can 2019; 41: 593-598. 
8. Wadhwa L, Rani P, Bhatia P. Comparative prospective study of hysterosalpingography and hysteroscopy in infertile women. $J$ Hum Reprod Sci 2017; 10: 73-78.

9. Fang F, Bai Y, Zhang Y, Faramand A. Oil-based versus water-based contrast for hysterosalpingography in infertile women: a systematic review and meta-analysis of randomized controlled trials. Fertil Steril 2018; 110: 153-160.e3.

10. Dreyer K, van Eekelen R, Tjon-Kon-Fat RI, van der Steeg JW, Steures P, Eijkemans M, et al. The therapeutic effect of hysterosalpingography in couples with unexplained subfertility: a post-hoc analysis of a prospective multi-centre cohort study. Reprod Biomed Online 2019; 38: 233-239.

11. Fukunaga T, Fujii S, Inoue C, Mukuda N, Murakami A, Tanabe $\mathrm{Y}$, et al. The spectrum of imaging appearances of müllerian duct anomalies: focus on MR imaging. Jpn J Radiol 2017; 35: 697-706.
12. Rikken JF, Kowalik CR, Emanuel MH, Mol BW, Van der Veen F, van Wely M, et al. Septum resection for women of reproductive age with a septate uterus. Cochrane Database Syst Rev 2017; 1: CD008576.

13. Ludwin A, Martins WP, Nastri CO, Ludwin I, Coelho Neto MA, Leitão VM, et al. Congenital Uterine Malformation by Experts (CUME): better criteria for distinguishing between normal/arcuate and septate uterus? Ultrasound Obstet Gynecol 2018; 51: 101-109.

14. Volondat M, Fontas E, Delotte J, Fatfouta I, Chevallier P, Chassang M. Magnetic resonance hysterosalpingography in diagnostic work-up of female infertility - comparison with conventional hysterosalpingography: a randomised study. Eur Radiol 2019; 29: 501-508.

15. Bhatt S, Sumbul M, Rajpal R, Radhakrishnan G. Value of "Three Dimensional Multidetector CT Hysterosalpingography" in infertile patients with non-contributory hysterosalpingography: A prospective study. J Reprod Infertil 2017; 18: 323-32.

\section{Ethical Consent}

All manuscripts reporting the results of experimental investigations involving human subjects should include a statement confirming that informed consent was obtained from each subject or subject's guardian, after receiving approval of the experimental protocol by a local human ethics committee, or institutional review board. When reporting experiments on animals, authors should indicate whether the institutional and national guide for the care and use of laboratory animals was followed. 